\title{
Evaluación estacional de la riqueza y abundancia de especies de mamíferos en la Reserva Biológica Municipal "Mário Viana", Mato Grosso, Brasil
}

\author{
Ednaldo Cândido Rocha ${ }^{1}$, Elias Silva ${ }^{2}$, Sebastião Venâncio Martins ${ }^{2}$ \& Francisco Cândido \\ Cardoso Barreto ${ }^{3}$ \\ 1 Pos-Graduación en Ciencia Forestal, Departamento de Ingeniería Forestal, Universidad Federal de Viçosa, Brasil. \\ CEP 36570-000; ednaldorocha@yahoo.com.br \\ 2 Departamento de Ingeniería Forestal, Universidad Federal de Vinosa, Brasil. CEP 36570-000. \\ 3 Pos-Graduación en Entomología, Departamento de Biología Animal, Universidad Federal de Vinosa, Brasil. CEP \\ $36570-000$
}

Recibido 17-VI-2005. Corregido 20-IV-2006. Aceptado 23-V-2006.

\begin{abstract}
Seasonal evaluation of mammal species richness and abundance in the "Mário Viana" municipal reserve, Mato Grosso, Brasil. We evaluated seasonal species presence and richness, and abundance of medium and large sized mammalian terrestrial fauna in the "Mário Viana" Municipal Biological Reserve, Nova Xavantina, Mato Grosso, Brazil. During 2001, two monthly visits were made to an established transect, $2820 \mathrm{~m}$ in length. Records of 22 mammal species were obtained and individual footprint sequences quantified for seasonal calculation of species richness and relative abundance index (x footprints $/ \mathrm{km}$ traveled). All 22 species occurred during the rainy season, but only 18 during the dry season. Pseudalopex vetulus (Lund, 1842) (hoary fox), Eira barbara (Linnaeus, 1758) (tayra), Puma concolor (Linnaeus, 1771) (cougar) and Hydrochaeris hydrochaeris (Linnaeus, 1766) (capybara) were only registered during the rainy season. The species diversity estimated using the Jackknife procedure in the dry season $(19.83, \mathrm{CI}=2.73)$ was smaller than in the rainy season (25.67, CI=3.43). Among the 18 species common in the two seasons, only four presented significantly different abundance indexes: Dasypus novemcinctus Linnaeus, 1758 (nine-banded armadillo), Euphractus sexcinctus (Linnaeus, 1758) (six-banded armadillo), Dasyprocta azarae Lichtenstein, 1823 (Azara's Agouti) and Tapirus terrestris (Linnaeus, 1758) (tapir). On the other hand, Priodontes maximus (Kerr, 1792) (giant armadillo) and Leopardus pardalis (Linnaeus, 1758) (ocelot) had identical abundance index over the two seasons. Distribution of species abundance in the sampled area followed the expected pattern for communities in equilibrium, especially in the rainy season, suggesting that the environment still maintains good characteristics for mammal conservation. The present study shows that the reserve, although only 470 ha in size, plays an important role for conservation of mastofauna of the area as a refuge in an environment full of anthropic influence (mainly cattle breeding in exotic pasture). Rev. Biol. Trop. 54 (3): 879-888. Epub 2006 Sept. 29.
\end{abstract}

Key words: mammals, seasonal evaluation, footprints, abundance and species richness.

De los mamíferos descritos actualmente, aproximadamente 524 especies se hallan en territorio brasileño, lo que representa cerca de $13 \%$ de la mastofauna del mundo. Estos números hacen que Brasil posea la mayor riqueza de mamíferos de toda la región neotropical (Fonseca et al. 1996). Los mamíferos presentes en el Cerrado totalizan cerca de 195 especies, de las cuales 18 son endémicas y 16 están incluidas en la lista nacional de especies de la fauna brasileña con amenaza de extinción (MMA 2003).

Los primeros registros sobre la mastofauna de la región de Nova Xavantina, este del estado de Mato Grosso, fueron realizados durante la expedición anglo-brasileña Xavantina-Cachimbo al final de la década de 1960 (Pine et al. 1970). Posteriormente, otros trabajos sobre mamíferos también fueron desarrollados en esa región (WWF 2000, Dalponte 2003). 
Datos sobre abundancia y distribución son muy importantes para la evaluación del estado de conservación de un taxón (IUCN 2001), siendo una de las dificultades para dicha evaluación en el ámbito nacional, la escasez de datos publicados sobre riqueza y abundancia de las especies en niveles locales y regionales. Además de esto, las informaciones sobre la distribución de las especies a lo largo del año son de gran importancia para la elaboración de un plan de manejo de unidades de conservación. Teniendo en cuenta el marco de referencia anterior, el objetivo de este trabajo fue realizar una evaluación estacional de la riqueza y abundancia de especies para la fauna de mamíferos terrestres de tamaño mediano y grande en la Reserva Biológica Municipal "Mario Viana" (RBMMV).

\section{MATERIALES Y MÉTODOS}

Área de estudio: El estudio fue realizado en la Reserva Biológica Municipal "Mario Viana”, RBMMV (1442' S y 52 $21^{\prime}$ ' W). Se trata de un fragmento de Cerrado que posee un área de aproximadamente 470 ha, se localiza a $5 \mathrm{~km}$ del centro de la ciudad de Nova Xavantina, donde se encuentra el Campus de la Universidad del Estado de Mato Grosso-UNEMAT.

El clima de la región es del tipo Aw, tropical estacional, caracterizado por dos estaciones bien definidas, una seca (de abril a septiembre) y una lluviosa (de octubre a marzo), según la clasificación de Köppen (Nimer 1989). La precipitación pluviométrica media anual es de $1500 \mathrm{~mm}$ y más del $90 \%$ de la precipitación ocurre de octubre a marzo (WWF 1995). El relieve es caracterizado de plano a ondulado, altitudes entre 250 y $300 \mathrm{~mm}$ y con presencia de suelo litólicos, cambissolo transicional con depósitos de arenito y latossolo rojo amarillento (RadamBrasil 1981).

La RBMMV posee fisionomías vegetales características de Cerrado sensu lato, con el predominio del Cerrado sensu stricto. Hay también formaciones como Cerradão, Campo
Limpio y Mata de Galería (Felfili et al. 1998, Marimon et al. 1998). Se han eliminado los impactos anteriores producidos por la extracción de madera y plantación de pasto exótico permitiendo un proceso de recuperación del área después de la implantación del Campus de la UNEMAT. En la actualidad el área ha sido fuente de varios proyectos de conservación de los medios físico y biótico.

Recolección de datos: Los datos fueron recolectados en el periodo de enero a diciembre del 2001, realizándose dos visitas mensuales a un transecto de $2820 \mathrm{~m}$ de extensión, totalizando $67680 \mathrm{~m}$ recorridos, durante 24 rastreos. El muestreo de mamíferos de tamaño mediano y grande, con peso por encima de $5 \mathrm{~kg}$ para los adultos (Hayward y Phillipson 1979), se realizó mediante levantamiento e interpretación de sus huellas (rastreos). En cada visita, el transecto fue rigurosamente inspeccionado, las huellas halladas fueron identificadas (Becker y Dalponte 1991) y la información fue organizada para obtener la abundancia relativa de las especies y sus índices de riqueza.

Para la evaluación de las secuencias de las huellas se tuvo presente que las mismas no fuesen tomadas en cuenta repetidamente durante el muestreo. Se utilizaron los siguientes criterios para la individualización de las mismas: la dirección tomada por el animal, el tamaño de la huella y la distancia entre las secuencias de huellas.

Los rastreos fueron hechos siempre por la mañana (a partir de 07:00 am) y cada uno tomó cerca de 1 h y $30 \mathrm{~min}$. Para evitar el recuento de rastros de los días anteriores, se procedió de la siguiente forma: (1) para la estación lluviosa, los rastreos se realizaron en la primera mañana después de una tarde de lluvia (Brower y Zar 1984); y (2) para la estación seca, las huellas viejas fueron borradas utilizando escobas, la tarde anterior al día de la evaluación.

A pesar de que algunas especies listadas en el presente estudio no sean de tamaño mediano y grande, debido a que no superan los $5 \mathrm{~kg}$ para los adultos, de acuerdo con Hayward y Phillipson (1979), se incluyeron debido a la seguridad que se tuvo para la identificación de sus huellas. 
Estas especies fueron: Didelphis albiventris (Lund, 1840) (comadreja overa), Dasypus novemcinctus (Linnaeus, 1758) (armadillo de nueve bandas), Pseudalopex vetulus (Lund, 1842) (zorro raposa de campo), Herpailurus yaguarondi (Lacépède, 1809) (yaguarundí), Leopardus tigrinus (Schreber, 1775) (tigrillo), Dasyprocta azarae (Lichtenstein, 1823) (agutí) y Sylvilagus brasiliensis (Linnaeus, 1758) (conejo silvestre) (Fonseca et al. 1996).

Análisis de datos: La identificación y evaluación de las huellas ha sido usada como un índice de abundancia, sobretodo para especies de difícil visualización o captura (Wilkie y Finn 1990, Nichols y Conroy 1996, Stander et al. 1997). Por lo tanto, algunos investigadores utilizan el registro de huellas en parcelas (plots) de arena previamente preparadas $\mathrm{y}$, muchas veces, utilizan carnadas para atraer los animales (Pardini et al. 2003, Scoss et al. 2004). Sin embargo, en este estudio se buscó una manera de presentar la abundancia relativa de las especies creando un índice individual de huellas por kilómetro recorrido, ya que el área dispone de un transecto continuo, con posibilidad de hacer rastreos en gran parte de su extensión.

A partir de los datos de abundancia de las muestras, en el transecto de cada estación (seca y lluviosa) fue calculada la riqueza de especies, por el procedimiento Jackknife (Heltshe y Forrester 1983, Heltshe y Forrester 1985, Hellmann y Fowler 1999), utilizándose el programa Estimates versión 7.0 (Colwell 2005). El método Jackknife estima la riqueza sumando la riqueza observada (el número de especies de la muestra) a un parámetro calculado a partir del número de especies raras y del número de muestras (Santos 2003). También se comparó la abundancia estacional de cada especie, por la prueba t-Student, generando gráficos de riqueza y de abundancia relativa de las especies en cada estación. Se utilizó el modelo log-normal para el ajuste de las curvas de la distribución de abundancia de las especies (May 1975).

\section{RESULTADOS}

Obtuvimos registros de 22 especies de mamíferos en el transecto muestreado (un marsupial, cinco xenartros, ocho carnívoros, un perisodáctilo, tres artiodáctilos, tres roedores y un lagomorfo), los cuales tuvieron sus secuencias individuales de huellas cuantificadas para el cálculo estacional de la riqueza de especies y del índice de abundancia relativa, dado por la media de secuencias individuales de huellas por kilómetro recorrido. De esas especies, cinco se hallan en amenaza de extinción (MMA 2003). Todas las especies se encontraron durante la estación lluviosa y apenas 18 de ellas fueron registradas durante la estación seca (Cuadro 1).

Estimación de riqueza y abundancia de especies: Se estimó la riqueza estacional de las especies, donde la estación seca presentó la menor riqueza (19.83, con intervalo de confianza $(\mathrm{IC})=2.73)$ que la estación lluviosa (25.67, con $\mathrm{IC}=3.43)$. Se destaca que para ambas estaciones, el muestreo fue suficiente para representar la comunidad de mamíferos en la área estudiada, pues las curvas de acumulación de especies se estabilizaron (Fig. 1 y 2).

Se realizó una comparación entre el índice de abundancia estacional para cada especie a fin de verificarse la existencia de algún patrón estacional. Entre las 18 especies comunes a las dos estaciones, 14 no presentaron diferencias significativas entre sus índices de abundancia (T-Student, $\mathrm{p}<.05$ ) y cuatro mostraron índices de abundancia significativamente diferentes: D. novemcinctus (armadillo de nueve bandas), Euphractus sexcinctus (Linnaeus, 1758) (armadillo de seis bandas), D. azarae (agutí), fueron más abundantes en la estación lluviosa, y Tapirus terrestris (Linnaeus, 1758) (tapir), con mayor abundancia en la estación seca. Priodontes maximus (Kerr, 1792) (armadillo gigante) y Leopardus pardalis (Linnaeus, 1758) (ocelote) se destacaron por presentar un índice de abundancia idéntico entre las dos estaciones (Cuadro 1). 


\section{CUADRO 1}

Índice de abundancia calculado a partir de la frecuencia estacional de las secuencias individuales de huellas de mamíferos por kilómetro recorrido, con sus respectivas desviaciones estándar, y comparación de la abundancia entre las estaciones seca y lluviosa. Los registros fueron efectuados en Reserva Biológica Municipal "Mário Viana", Nova Xavantina-Mato Grosso, Brasil, en el periodo de enero a diciembre de 2001

\section{TABLE 1}

Abundance index calculated from the seasonal frequency of individual sequences of mammal footprints per kilometer traveled, with their respective standard deviation, and comparison of abundance between dry and rainy seasons.

The recordings were made in the Municipal Biological Reserve "Mário Viana",

Nova Xavantina - Mato Grosso, Brazil, from January to December in 2001

Orden/Familia/Especie

Didelphimorphia

Didelphidae

Didelphis albiventris Lund, 1840

Xenarthra

Myrmecophagidae

Tamandua tetradactyla (Linnaeus, 1758)

Dasypodidae

Dasypus novemcinctus Linnaeus, 1758

Euphractus sexcinctus (Linnaeus, 1758)

Priodontes maximus (Kerr, 1792)*

Carnivora

Canidae

Cerdocyon thous (Linnaeus, 1766)

Pseudalopex vetulus (Lund, 1842)

Procyonidae

Procyon cancrivorus (G. Cuvier, 1798)

Mustelidae

Eira barbara (Linnaeus, 1758)

Felidae

Herpailurus yaguaroundi (Lacépède, 1809)
Leopardus pardalis (Linnaeus, 1758)*
Leopardus tigrinus (Schreber, 1775)*
Puma concolor (Linnaeus, 1771)*

Perissodactyla

Tapiridae

Tapirus terrestris (Linnaeus, 1758)

Artiodactyla
Índice de

abundancia

Estación seca

$0.68 \pm 0.59$

$0.18 \pm 0.24$

$0.50 \pm 0.49$

$0.15 \pm 0.18$

$0.03 \pm 0.10$

$0.38 \pm 0.24$

$0.21 \pm 0.24$

$-1.832$

0.081

$0.06 \pm 0.14$

$0.03 \pm 0.10$

$0.06 \pm 0.14$

0.596

0.557

$0.06 \pm 0.14$

$0.06 \pm 0.14$

$0.12 \pm 0.23$

$0.09 \pm 0.16$

$0.09 \pm 0.16$

0.484

0.633

$0.12 \pm 0.23$

0.000

1.000

$0.18 \pm 0.19$

1.254

0.223

$0.03 \pm 0.10$

$0.35 \pm 0.26$

$0.12 \pm 0.17$

$-2.602$

0.016 
CUADRO 1 (Continuación)

Índice de abundancia calculado a partir de la frecuencia estacional de las secuencias individuales de huellas de mamíferos por kilómetro recorrido, con sus respectivas desviaciones estándar, y comparación de la abundancia entre las estaciones seca y lluviosa. Los registros fueron efectuados en Reserva Biológica Municipal "Mário Viana", Nova Xavantina-Mato Grosso, Brasil, en el periodo de enero a diciembre de 2001

TABLE 1 (Continued)

Abundance index calculated from the seasonal frequency of individual sequences of mammal footprints per kilometer traveled, with their respective standard deviation, and comparison of abundance between dry and rainy seasons.

The recordings were made in the Municipal Biological Reserve "Mário Viana",

Nova Xavantina - Mato Grosso, Brazil, from January to December in 2001

Orden/Familia/Especie $\quad \begin{gathered}\text { Índice de } \\ \text { abundancia }\end{gathered} \quad \begin{gathered}\text { Comparación entre } \\ \text { las estaciones }\end{gathered}$

Tayassuidae

Pecari tajacu (Linnaeus, 1758)

$0.92 \pm 1.12$

$0.77 \pm 1.01$

$-0.340$

0.737

Cervidae

Mazama americana (Erxleben, 1777)

$0.09 \pm 0.16$

$0.12 \pm 0.23$

0.364

0.719

Mazama gouazoupira (G. Fischer, 1814)

$0.59 \pm 0.41$

$0.65 \pm 0.25$

0.425

0.675

Rodentia

Hydrochaeridae

Hydrochaeris hydrochaeris (Linnaeus, 1766)

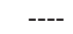

$0.03 \pm 0.10$

Agoutidae

Agouti paca (Linnaeus, 1766)

$0.21 \pm 0.24$

$0.09 \pm 0.16$

$-1.431$

0.167

Dasyproctidae

Dasyprocta azarae Liechtenstein, 1823

$1.86 \pm 0.64$

$2.66 \pm 0.63$

3.062

0.006

Lagomorpha

Leporidae

Sylvilagus brasiliensis (Linnaeus, 1758)

$1.24 \pm 0.39$

$1.33 \pm 0.59$

0.437

0.667

*Especies amenazadas de extinción (MMA 2003).

Los valores en negrita indican diferencias significativas por la prueba T-Student, $\mathrm{p}<.05$

\section{DISCUSIÓN}

Estimación de la riqueza: La riqueza de especies estimada en ambas estaciones, seca $(19.83$, con $\mathrm{IC}=2.73)$ y lluviosa $(25.67$, con $\mathrm{IC}=3.43$ ), fue próxima a la riqueza observada con 18 y 22 especies, respectivamente. Estas informaciones indican que el muestreo representó muy bien a la comunidad de mamíferos estudiados en la RBMMV, en ambas las estaciones. Según Santos (2003), utilizando el procedimiento Jackknife, cuando todas las especies observadas aparecen en más de una muestra, la riqueza estimada es igual a la observada, es decir, el método considera que todas las especies del ambiente fueran muestreadas. Este hecho también se observa cuando las curvas de acumulación de especies se estabilizan rápidamente (Fig. 1).

En cuanto a las especies que fueron registradas exclusivamente en la estación lluviosa, $P$. vetulus posiblemente no fue bien representada en el muestreo, pues esta especie vive prioritariamente en hábitats abiertos (Dalponte 

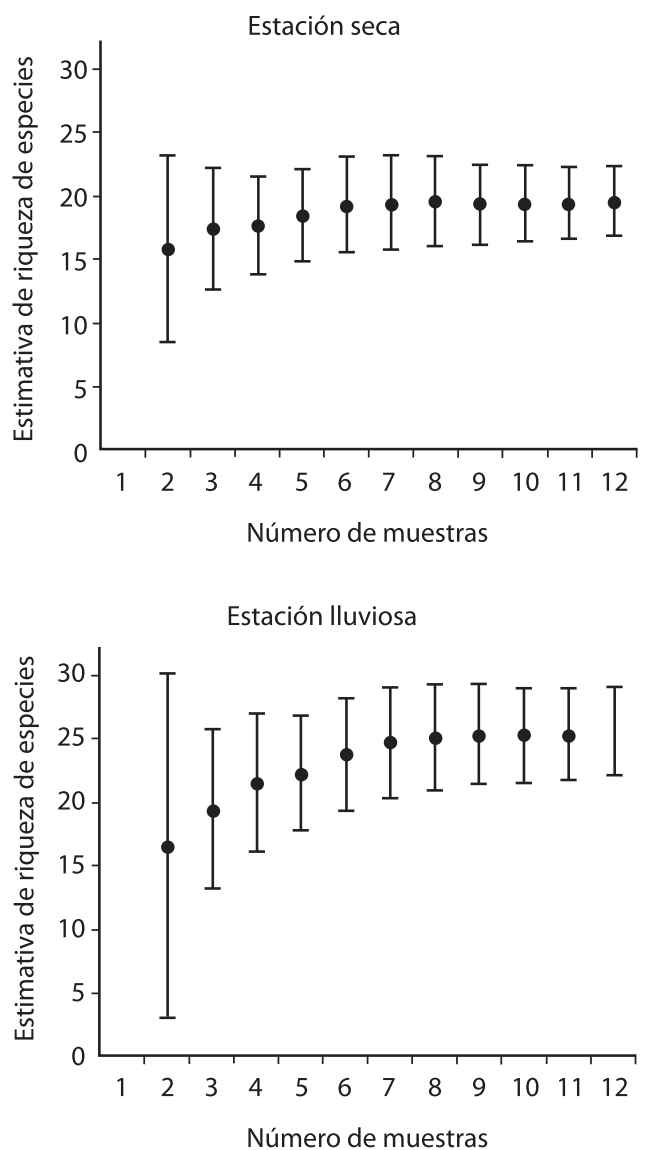

Fig. 1. Estimación de riqueza de especies calculada mediante el procedimiento Jackknife a partir del registro estacional de huellas de mamíferos en la Reserva Biológica Municipal "Mário Viana", Nova Xavantina-Mato Grosso, Brasil, en el periodo de enero a diciembre de 2001. Las barras indican el intervalo de confianza $(\mathrm{p}<.05)$.

Fig. 1. Species richness estimation via Jackknife procedure using the seasonal recording of mammal footprints in the Municipal Biological Reserve "Mário Viana", Nova Xavantina-Mato Grosso, Brazil, from January to December 2001.Bars indicate the confidence interval $(\mathrm{p}<.05)$.

1997, 2003), que no son comunes en el área comprendida para el transecto. Por lo tanto, el índice de abundancia de esta especie pudo haber sido subestimado y la ausencia de registro en la estación seca no se debe al patrón estacional, siendo que la mayor abundancia de $P$. vetulus en la RBMMV, en ambas estaciones, podrá ser encontrada en áreas abiertas por acción antrópica. Eira barbara (Linnaeus,

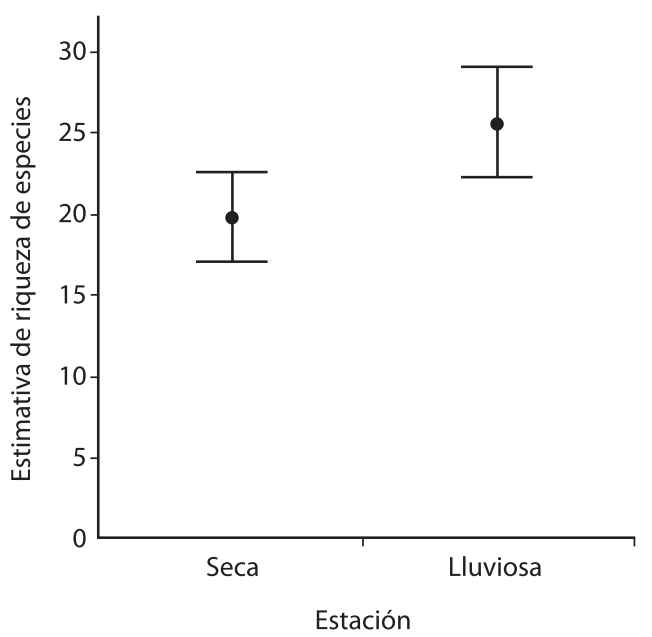

Fig. 2. Comparación de la estimación de riqueza estacional de especies de mamíferos registrados en la Reserva Biológica Municipal "Mário Viana", Nova XavantinaMato Grosso, Brasil, en 2001. Las barras indican el intervalo de confianza $(\mathrm{p}<.05)$.

Fig. 2. Comparison of seasonal richness estimation of mammal species recorded in the Municipal Biological Reserve "Mário Viana", Nova Xavantina-Mato Grosso, Brazil, in 2001. Bars indicate the confidence interval $(p<.05)$.

1758) (hurón mayor), fue registrada apenas en el último mes de muestreo (diciembre 2001), lo que no caracterizó el patrón estacional de actividad. Además de esto, no se descarta la posibilidad de que esta especie sea cazada por moradores del entorno de la RBMMV, ya que ella ocasionalmente puede alimentase de aves domésticas y entrar en plantaciones, aunque los daños son mínimos (Nowak 1999). Puma concolor (Linnaeus, 1771) (puma) y Hydrochaeris hydrochaeris (Linnaeus, 1766) (capibara) posiblemente no viven en la RBMMV, apenas utilizaron el área de tránsito o como refugio durante algunos de sus desplazamientos. Esto se debe al hecho que la reserva no dispone de un área con tamaño suficiente para mantener un depredador de la cima de la cadena alimentaría como $P$. concolor, y también por que ésta área no posee un hábitat adecuado para la presencia de H. hydrochaeris (Marinho-Filho et al. 1998), pues solamente presenta apenas un arroyo estacional. 


\begin{abstract}
Abundancia de especies: La abundancia
\end{abstract} significativamente mayor de D. novemcinctus, E. sexcinctus y D. azarae en la estación lluviosa puede estar relacionados con una mayor disponibilidad de recursos en el medio ambiente, una vez que en este periodo el crecimiento de la vegetación es favorecido, pues el suelo queda húmedo y hay mayor disponibilidad de recursos hídricos. Además de esto en la estación lluviosa los rastreos acontecieron siempre en la primera mañana después de una tarde de lluvia, cuando la temperatura y la humedad parecen adecuadas para la actividad de $D$. novemcinctus y E. sexcinctus. Una posible explicación para este hecho es que la actividad máxima de $E$. sexcinctus ocurre cuando la temperatura oscila entre $20-25^{\circ} \mathrm{C}$, siendo que ese animal se muestra más activo en el periodo nocturno durante el verano (Nowak 1991). Por otro lado, en áreas habitadas por esta especie es relativamente común encontrarla forrajeando en la tarde, inmediatamente después del término de alguna lluvia (E.C. Rocha, obs. pers.). Para D. novemcinctus, la temperatura y no la luminosidad parece ser la señal de actividad e inactividad de la especie (Fox 1999). En el caso de D. azarae, se debe resaltar que la metodología de rastreos en transectos continuos, puede no ser exacta para estimar su abundancia, pues el patrón difuso de movilización de esa especie dificulta la individualización de sus secuencias de huellas, especialmente en áreas donde se encuentra una alta abundancia como en la RBMMV.

El hecho de $T$. terrestris presente una abundancia significativamente mayor en la estación seca, puede estar relacionado con la dieta de la especie, que es estrictamente vegetariana. En esta estación el animal tiene que desplazarse más para encontrar suficiente cantidad de alimento. Otra posible explicación de la abundancia de $T$. terrestris, es que esta época puede coincidir con la época de apareamiento.

Otros factores que no fueron evaluados, pero que pueden haber influenciado la abundancia de las especies en la RBMMV son: presencia humana, distribución de la precipitación pluviométrica en cada estación y desplazamientos de los animales durante el apareamiento, lactancia y dispersión de jóvenes.

En lo que se refiere a la distribución de abundancia relativa de las especies en el ambiente, una característica que marca las comunidades en general es que ellas tienen, comparativamente, pocas especies que son abundantes y muchas especies que son raras (Krebs 1999). Éste es un patrón encontrado comúnmente en grupos de datos que se ajustan a la distribución log-normal (Fig. 3), lo que indica que la comunidad fue bien muestreada y que la misma presenta una razonable complejidad de interacciones. En condiciones de equilibrio, una distribución log-normal de la abundancia relativa de las especies tiene mayor probabilidad de ocurrir, debido a que la comunidad posee un número de especie relativamente elevado que cumple diversas funciones en el ambiente (May 1975). Debido a la distribución de la abundancia de las especies de mamíferos registradas en la RBMMV, se puede deducir que el área estudiada, aunque es pequeña (aproximadamente de $470 \mathrm{ha}$ ) y presenta algunos disturbios accidentales (como incendios, presencia de animales domésticos y hasta la propia caza), presentó una comunidad de mamíferos relativamente diversa y bien distribuida en términos de abundancia relativa, especialmente en la estación lluviosa. La metodología utilizada para este estudio fue adecuada, pues generó resultados representativos sobre la riqueza y la abundancia relativa de la mastofauna en la RBMMV y puede ser empleada en estudios de monitoreo de comunidades de mamíferos de medio y gran tamaño.

Para las condiciones encontradas en la RBMMV, en el periodo de estudio, la estación lluviosa es más rica en especies que la estación seca, ocurriendo que cuatro especies fueran registradas exclusivamente en el periodo lluvioso: P. vetulus, E. barbara, P. concolor y $H$. hydrochaeris. En forma general, no hubo diferencia significativa en la abundancia estacional de registros de huellas de las especies de mamíferos en la RBMMV, pues apenas cuatro 

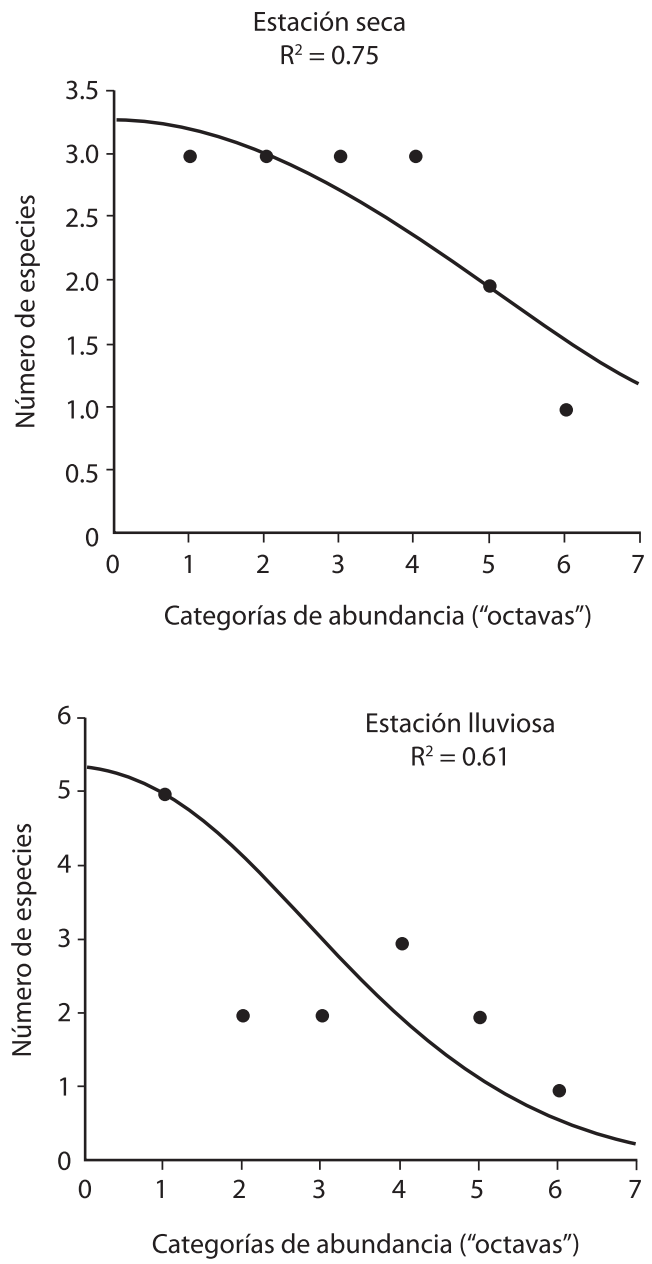

Fig. 3. Curvas log-normales de la distribución de la abundancia relativa de las especies, ajustadas a partir del registro estacional de huellas de mamíferos en la Reserva Biológica Municipal "Mário Viana", Nova Xavantina-Mato Grosso, Brasil, en el periodo de enero a diciembre de 2001.

Fig. 3. Log-normal distribution of relative species abundance, adjusted from the seasonal recordings of mammal footprints in the Municipal Biological Reserve "Mário Viana", Nova Xavantina-Mato Grosso, Brazil, from January to December 2001.

especies presentaron diferencias significativas en sus índices de abundancia entre las estaciones: D. novemcinctus, E. sexcinctus, D. azarae y $T$. terrestris.

La distribución de la abundancia de las especies sigue aproximadamente el patrón esperado para comunidades en equilibrio, evidenciando que el ambiente aún mantiene buena calidad para la conservación de los mamíferos. A pesar de que RBMMV tenga un área pequeña, desempeña un papel importante en la conservación de la mastofauna de la región de Nova Xavantina, por ser un área de refugio en un ambiente con mucha influencia antrópica, principalmente por la creación de ganado en pasto exótico.

\section{AGRADECIMIENTOS}

A Rogério Cândido Rocha y Tito Bacca por sus importantes comentarios y revisión de este trabajo.

\section{RESUMEN}

Estudiamos la fauna de mamíferos terrestres medianos y grandes, tomando en cuenta la riqueza y abundancia de las especies y la cantidad de individuos, en la Reserva Biológica Municipal "Mário Viana", Nova Xavantina, Mato Grosso, Brasil. Hicimos dos visitas mensuales durante todo el año 2001 en un transecto de 2820 m de extensión, previamente preparado para la identificación de huellas. Identificamos 22 especies en la estación lluviosa y 18 de ellas también en la seca. Registramos Pseudalopex vetulus (Lund, 1842) (zorro), Eira barbara (Linnaeus, 1758) (hurón), Puma concolor (Linnaeus, 1771) (puma) e Hydrochaeris hydrochaeris (Linnaeus, 1766) (capiguara) durante la estación de lluvias. Según el procedimiento Jackknife, la riqueza de especies durante la estación seca $(19.83$, con intervalo de confianza $($ IC $)=2.73)$ fue menor que durante la estación lluviosa $(25.67$, con $\mathrm{IC}=$ 3.43). Solamente cuatro mostraron índices de abundancia significativamente diferentes entre estaciones: Dasypus novemcinctus Linnaeus, 1758 (armadillo de nueve bandas), Euphractus sexcinctus (Linnaeus, 1758) (armadillo de seis bandas), Dasyprocta azarae Lichtenstein, 1823 (agutí) y Tapirus terrestris (Linnaeus, 1758) (tapir). Por otro lado, Priodontes maximus (Kerr, 1792) (armadillo gigante) y Leopardus pardalis (Linnaeus, 1758) (ocelote) se destacaron por presentar un índice de abundancia idéntico entre las estaciones. La distribución de la abundancia de las especies en el área de muestreo, siguió más o menos el patrón esperado para las comunidades en equilibrio, especialmente en la estación lluviosa, evidenciando que el ambiente aún mantiene una buena calidad para la conservación de los mamíferos. El presente estudio mostró que la RBMMV, a pesar de ser pequeña (con aproximadamente $470 \mathrm{ha}$ ), desempeña un papel importante para la conservación de la mastofauna de la región, siendo un área de refugio en un 
ambiente con mucha influencia antrópica, principalmente por la cría de ganado con pasto exótico.

Palabras clave: mamíferos, evaluación estacional, huellas, abundancia y riqueza de especies.

\section{REFERENCIAS}

Becker, M. \& J.C. Dalponte. 1991. Rastros de mamíferos silvestres brasileiros: una guía de campo. Universidade de Brasília, Brasília. 180 p.

Brower, J.E. \& J.H. Zar. 1984. Field \& laboratory methods for General Ecology. Wm. C. Brown, Dubuque, Iowa, EEUU. 226 p.

Dalponte, J.C. 1997. Diet of the hoary fox, Lycalopex vetulus, in Mato Grosso, Central Brazil. Mammalia 61: 537-546.

Dalponte, J.C. 2003. História natural, comportamento e conservação da raposa-do-campo, Pseudalopex vetulus (Canidae). Tesis de doctorado, Universidade de Brasília, Brasília, Brasil. 179 p.

Felfili, J.M., M.C. Silva-Junior \& P.E. Nogueira. 1998. Levantamento da vegetação arbórea na região de Nova Xavantina, MT. Boletim do Herbário Ezechias Paulo Heringer 3: 63-81.

Fonseca, G.A.B., G. Herrmann, Y.L.R. Leite, R.A. Mittermeier, A.B. Rylands \& J.L. Patton. 1996. Lista Anotada dos Mamíferos do Brasil. Conservation Biology, v. 4 (Occasional Papers). 38 p.

Hayward, G.F. \& J. Phillipson. 1979. Community structure and functional role of small mammals in ecosystems, p. 135-211. In D.M. Stoddart (ed.). Ecology of small mammals. Chapman and Hall, Londres, Inglaterra.

Hellmann, J.J. \& G.W. Fowler. 1999. Bias, precision, and accuracy of four measures of species richness. Ecol. Appl 9: 824-834.

Heltshe, J.F. \& N.E. Forrester. 1983. Estimating species richness using the jackknife procedure. Biometrics 39: 1-11.

Heltshe, J. F. \& N.E. Forrester. 1985. Statistical evaluation of the jackknife estimate of diversity when using quadrat samples. Ecology 66: 107-111.

IUCN. 2001. Red List Categories and Criteria: version 3.1. IUCN-Species Survival Commission. Gland, Suiza. 32 p.

Krebs, C.J. 1999. Ecological Methodology. AddisonWelsey Educational, California, EEUU. 620 p.
Marimon, B.S., R.F. Varella \& B.H. Marimon-Júnior. 1998. Fitossociologia de uma área de cerrado de encosta em Nova Xavantina, Mato Grosso. Boletim do Herbário Ezechias Paulo Heringer 3: 82-101.

Marinho-Filho, J., F.H.G. Rodrigues, M.M. Guimarães \& M.L. Reis. 1998. Os mamíferos da Estação Ecológica de Águas Emendadas, Planaltina, DF, p. 34-63. In J. Marinho-Filho, F.H.G. Rodrigues \& M.M. Guimarães (eds.). Vertebrados da Estação Ecológica de Águas Emendadas-História Natural e Ecologia em um fragmento de Cerrado do Brasil Central. SEMATEC/ IEMA, Brasilia.

May, R.M. 1975. Patterns of Species Abundance and Diversity, p. 81-120. In M.L. Cody \& J.M. Diamond (eds). Ecology and Evolution of Communities. Belknap, Cambridge, Inglaterra.

MMA-Ministério do Meio Ambiente. 2003. Espécies da Fauna Brasileira Ameaçadas de Extinção. Instrução Normativa $\mathrm{n}^{\circ} 3$, de 27 de maio de 2003. Publicación en el Diario Oficial de la Unión $\mathrm{n}^{\circ} 101$, en 28 de mayo de 2003, Sección 1, p. 88-97.

Nichols, J.D. \& M.J. Conroy. 1996. Techniques for Estimating Abundance and Species Richness, p. 177234. In D.E. Wilson, F.R. Cole, J.D. Nichols, R. Rudran \& M.S. Foster (eds.). Measuring and Monitoring Biological Diversity: Standard Methods for Mammals. Smithsonian Institution, Washington DC. EEUU.

Nimer, E. 1989. Climatologia do Brasil. IBGE, Rio de Janeiro, Brasil. 422 p.

Nowak, R.M. 1991. Walker's mammals of the World. Vol. I. Johns Hopkins University, Baltimore, Maryland. 642 p.

Pardini, R., E.H. Ditt, L.Jr. Cullen, C. Bassi \& R. Rudran. 2003. Levantamento rápido de mamíferos terrestres de médio e grande porte, p. 181-201. In L.Jr. Cullen, R. Rudran \& C. Valladares-Padua (Orgs). Métodos de estudos em Biologia da Conservação e Manejo da Vida Silvestre. Universidade Federal do Paraná, Curitiba, Brasil.

Pine, R.H., I.R. Bishop \& R.J. Jackson. 1970. Preliminary list of mammals of the Xavantina/Cachimbo expedition (Central Brazil). Trans. R. Soc. Trop. Med. Hyg. 64: 668-670.

RadamBrasil. 1981. Levantamento dos Recursos Naturais. Ministério das Minas e Energia: 25, folha SD-22 Goiás, Brasil.

Santos, A.J. 2003. Estimativas de riqueza em espécies, p. 19-41. In L.Jr. Cullen, R. Rudran \& C. Valladares-Padua (orgs). Métodos de estudos em Biologia da Conservação e Manejo da Vida Silvestre. Universidade Federal do Paraná, Curitiba, Brasil. 
Scoss, L.M., P.Jr. De Marco, E. Silva \& S.V. Martins. 2004. Uso de parcelas de areia para o monitoramento de impacto sobre a riqueza de especies de mamíferos. Rev. Árvore 28: 121-127.

Stander, P.E., D. Ghau, D. Tsisaba, Oma \& I.I. Ui. 1997. Tracking and the interpretation of spoor: a scientifically sound method in ecology. J. Zool. 242: 329-341.

Wilkie, D.S. \& J.T. Finn. 1990. Slash-burn Cultivation and Mammal Abundance in the Ituri Forest, Zire. Biotropica 22: 90-99.

WWF-Fundo Mundial para a Natureza. 1995. De grão em grão, o Cerrado perde espaço (Cerrado-Impacatos do processo de ocupação). Brasília-DF. 66 p.
WWF-Fundo Mundial para a Natureza. 2000. Manejo de Fauna na Reserva Xavante Rio das Mortes, MT. Brasilia. 64 p.

\section{REFERENCIAS DE INTERNET}

Colwell, R.K. 2005. Estimates: Statistical estimation of species richness and shared species from samples. Versión 7.0. (Consultado: 10 de abril de 2005, http:// viceroy.eeb.uconn.edu/estimates).

Fox, D. 1999. Dasypus novemcinctus, Animal Diversity Web. (Consultado: 25 de abril de 2005, http://www. animaldiversity.ummz.umich.edu/site/accounts/information/Dasypus_novemcinctus.html). 